\title{
Enfermedades ocupacionales del personal de enfermería por desempeño de labores en turnos rotativos y la importancia de los protocolos ergonómicos de prevención \\ Occupational diseases of nursing staff due to the performance of work in rotating shifts and the importance of ergonomic prevention protocols
}

\author{
María José Rueda Manzano ${ }^{1}$ \\ mrueda@puce.edu.ec \\ Miryam Toapanta \\ mtoapanta@puce.edu.ec \\ Ximena Andino \\ xandino@puce.edu.ec \\ María Luisa Rueda \\ Mrueda078@puce.edu.ec
}

Recibido: 1/07/2018, Aceptado: 1/09/2018

\begin{abstract}
RESUMEN
Las demandas de una sociedad en crecimiento, globalizada y cada vez más afectada por diferentes sintomatologías de salud, requiere de profesionales comprometidos y dispuestos a ofrecer sus servicios a otros, lo interesante de esto es la contradicción que se presenta cuando estos servidores, profesionales enfermeros que disponen de su tiempo y competencias para ayudar a mejor las condiciones de salud de otros, lo hacen bajo condiciones laborales, disposiciones de las prácticas de su profesión y costumbres operativas que van en detrimento de su propia salud, razón por la cual las autoras presentan este trabajo que tiene como objetivo general estudiar las enfermedades ocupacionales del personal de enfermería que labora por turnos rotativos en el área de Traumatología del Hospital Eugenio Espejo con la finalidad de establecer mecanismos o protocolos ergonómicos para su prevención, partiendo de un enfoque cuantitativo, diseño de campo, tipo descriptivo, planteando como técnica de recolección de la información una encuesta que se aplica a una población constituida por 13 profesionales de enfermería que laboran en el área 1 y 2 de Hospitalización de Traumatología del Hospital de especialidades Eugenio Espejo. El cuestionario se divide en tres importantes dimensiones: estado de salud, turnos nocturnos y cuestionario Maslach. Dentro de las principales alteraciones se encontraron trastornos músculo-esqueléticos, dolores de espalda alta, dolor en la muñeca, en miembros inferiores como rodillas, presentan cansancio emocional y despersonalización. Se recomienda principalmente la reorganización de los sistemas de trabajo y la implementación de mejores condiciones laborales.
\end{abstract}

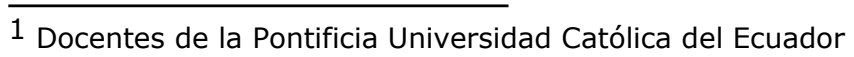


사

No. 20, 31 de octubre de 2018

ISSN impreso: 1390 - 6321

Palabras Claves: turnos, rotación, enfermedades ocupacionales, ergonomía

\begin{abstract}
The demands of a growing society, globalized and increasingly affected by different health symptoms, requires professionals committed and willing to offer their services to others, the interesting thing about this is the contradiction that occurs when these servers, nurses professionals they have their time and their skills to help improve the health conditions of others, they do it under working conditions, dispositions of their profession practices and operating customs that take into account their own health, reason for which the authors present a research work that has as its general objective; To study the occupational diseases of the nursing staff working in rotating shifts in the Traumatology area of the Eugenio Espejo Hospital with the purpose of establishing ergonomic mechanisms or protocols for their prevention, starting from a quantitative approach, field design, descriptive type, posing as information collection technique a survey that is applied to a population consisting of thirteen (13) nursing professionals who work in area 1 and 2 Hospitalization of Traumatology Hospital Specialties Eugenio Espejo, the questionnaire is divided into three important dimensions; health status, night shifts and Maslach questionnaire. Among the main alterations were musculoskeletal disorders, high back pain, pain in the wrist, lower limbs such as knees, emotional exhaustion and depersonalization. It is recommended mainly the reorganization of work systems and the implementation of better working conditions.
\end{abstract}

Keywords: shifts, rotation, occupational diseases, ergonomics

\title{
Introducción
}

El desempeño de la profesión de enfermería por su naturaleza, posee implicaciones en términos de salud y seguridad en el trabajo que exige un esfuerzo físico y mental en horarios biológicamente contradictorios, entre ellos, el establecimiento del sistema de trabajo rotativo, que se ha convertido en una actividad tradicional, una práctica frecuente y por tanto una característica particular y formalmente establecida de esta profesión, teniendo repercusión directa en la vida diaria de los trabajadores, al afectar no sólo su salud, sino también su calidad de vida ya que modifica actividades como el esparcimiento, las relaciones familiares y sociales (Cuartero, 2002).

Este tipo de trabajo conlleva unos riesgos para la salud y debe tratar de organizarse teniendo en cuenta sus implicaciones sobre esta, tanto a nivel físico, como psicológico o de interacción social... La vigilancia de los trastornos que se originan o agravan por este tipo de trabajos, la información o formación tanto a los trabajadores sometidos a ellos como a los empresarios también pueden mejorar de forma notable los efectos negativos que se dan en estas situaciones (p.25).

En el mismo orden de ideas, el trabajo nocturno, incluido como parte elemental de la planificación de los horarios en el trabajo rotativo origina en el individuo desincronización de sus funciones corporales, pues lo natural es el descanso en horas nocturnas, por lo que la alteración del ritmo circadiano tiene una relación contundente en la repercusión en la salud, generando enfermedades que van desde el estrés, fatiga crónica, trastornos osteomusculares, trastornos metabólicos y trastornos reproductivos (aborto), hasta enfermedades cardiovasculares, enfermedades 166 


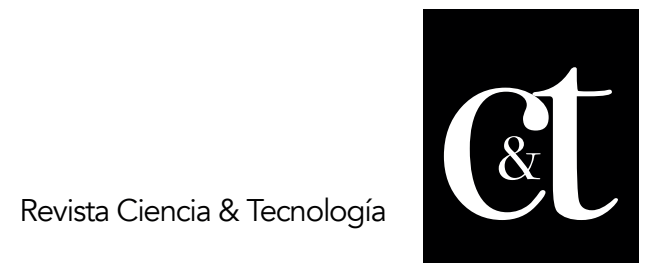

No. 20, 31 de octubre de 2018

ISSN impreso: 1390 - 6321

digestivas por la imposibilidad de comer en horarios regulares, la falta de apetito y la comida poco saludable (Miró, Cano y Buela, 2005).

Los seres humanos, al igual que otros seres vivos poseen un reloj corporal interno situado en el núcleo supraquiasmático del hipotálamo que regula sus ritmos corporales, incluido el ritmo de sueño-vigilia. El periodo habitual de la mayoría de estos ritmos es cercano a 24 horas (circadiano) y está preparado para sincronizarse con el ritmo ambiental diario de luz-oscuridad, en concreto, para estar activos y despiertos de día y para dormir y descansar de noche, coincidiendo con el descenso de la temperatura corporal que comienza a última hora del día. Cuando se trabaja de noche o en turnos rotativos de trabajo, el sistema circadiano es incapaz de adaptarse rápidamente al nuevo horario y surge una disincronía entre el ritmo de los sistemas fisiológicos internos y las exigencias horarias externas. Dicha disincronía, junto con la falta de sueño que suele asociarse a estas condiciones laborales, es responsable de los problemas de salud que se encuentran en este tipo de trabajadores (p. 18).

De manera que, en los últimos años, estos riesgos potenciales se han elevado rápidamente, una extensa serie de factores ambientales, físicos, y psicosociales potencialmente dañinos, amenazan constantemente al personal que desempeña labores en enfermería, aunado a ello, la actividad preventiva en los trabajadores sanitarios es escasamente vigilada y regulada, por lo que las autoras se motivan a vislumbrar a través del estudio que se presenta, las implicaciones que conciernen tanto en la teoría como en la práctica de las enfermedades ocupacionales del personal de enfermería por desempeño de labores en turnos rotativos y la importancia de los protocolos ergonómicos de prevención.

Por tanto, esta investigación inicia con una revisión documental que permitirá adentrarse a las diferentes variables que lo componen, así como las diversas posturas respecto al tema, luego se realiza un análisis a través de la recolección respectiva de los datos en una población finita de un grupo de profesionales de la enfermería, ubicados en el área 1 y 2 de Traumatología del Hospital Eugenio Espejo, quienes laboran en turnos rotativos de 12 horas diurnas, al siguiente día 12 horas nocturnas, con descanso de 72 horas, repitiendo el ciclo consecutivamente, debido a esto y otros hallazgos encontrados como observadores del objeto de estudio, las investigadoras parten de una pregunta a la cual pretenden dar respuesta en el transcurso y desarrollo del tema, ¿Cuáles son las enfermedades ocupacionales del personal de enfermería que labora por turnos rotativos en el área de Traumatología del Hospital Eugenio Espejo y que importancia tienen los protocolos ergonómicos para su prevención?.

Por consiguiente, se plantea como objetivo general; Estudiar las enfermedades ocupacionales del personal de enfermería que labora por turnos rotativos en el área de Traumatología del Hospital Eugenio Espejo con la finalidad de establecer mecanismos o protocolos ergonómicos para su prevención, se presenta bajo una estructura adecuada al enfoque de investigación en que se encuentra enmarcado el trabajo y las especificaciones propias de presentación como artículo científico, iniciando con la importancia del problema, donde se establece una descripción de las fundamentaciones teóricas, observaciones e información de la situación problemática que se presenta.

Rueda, Toapanta, Andino, Rueda. Enfermedades ocupacionales del personal de enfermería por desempeño de labores en turnos rotativos y la importancia de los protocolos ergonómicos de prevención 


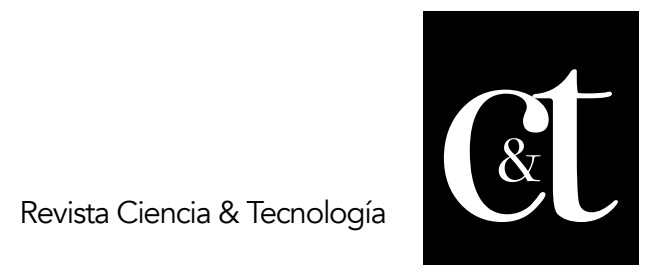

No. 20, 31 de octubre de 2018

ISSN impreso: 1390 - 6321

En el mismo orden de ideas se continua con la metodología; donde se explican los objetivos que direccionan el estudio, el enfoque, diseño y tipo de investigación, la población, técnicas e instrumentos para la recolección de los datos y la forma en que estos son analizados y presentados, luego se continua con la presentación de los resultados y su respectiva discusión, donde las autoras muestran los resultados de la aplicación del instrumento de recolección de la información, explican los más resaltantes o los hallazgos encontrados y se analizan, interpretan y discuten respecto a la teoría o fundamentaciones que preceden el estudio.

Para finalmente, concluir con las recomendaciones, y las fundamentaciones finales de la investigación que se presenta, conjuntamente con la lista de referencias bibliográficas que sirvieron de apoyo en el transcurso del desarrollo de este estudio, con la finalidad que dé respuesta a un protocolo ergonómico idóneo dirigido al personal o la población que se está estudiando, que puede contribuir o extenderse a beneficiar otros profesionales del sector salud que se encuentren en iguales 0 similares condiciones laborales, así mismo, como fuente de apoyo o de sustentación teórica para otras investigaciones que se asocien o refieran a las variables que se encuentran involucradas en el tema.

Importancia del problema

Según la Organización Mundial de la Salud, en adelante (OMS), cada año se producen alrededor de 1.2 millones de enfermedades profesionales en todo el mundo que son atribuibles por exposición o riesgos en trabajos peligrosos, esta carga elevada es la que más influye en la situación sanitaria de la población mundial porque al afectar en la salud de la población activa repercutirá decisivamente en la productividad y en el bienestar económico y social de los trabajadores del sector estructurado o no estructurado, sus familias y de las personas a su cargo. Las enfermedades ocupacionales son todas las alteraciones de la salud que se producen por exposición a factores de riesgo existentes en los ambientes de trabajo, de evolución aguda o crónica que lleva a incapacidad permanente y según su intensidad hasta la muerte.

En vista de la importancia que ha cobrado la salud en el trabajo, producto de la evolución de las concepciones y paradigmas que acompañan esta importante actividad humana, cada nación posee legislaciones que regulan los aspectos relacionados con la higiene, seguridad y salubridad en el trabajo, la Organización Internacional del Trabajo (1990, p. 3), en adelante OIT en el Convenio 171, Artículo 3 , expresa que se deberán adoptar en beneficio de los trabajadores nocturnos las medidas específicas requeridas por la naturaleza del trabajo nocturno... a fin de proteger su salud, ayudarles a cumplir con sus responsabilidades familiares y sociales, proporcionarles posibilidades de mejoras en su carrera y compensarles adecuadamente. Tales medidas deberán también tomarse en el ámbito de la seguridad y de la protección de la maternidad, en favor de todos los trabajadores que realizan un trabajo nocturno. Encontramos en la Constitución de la República del Ecuador (Asamblea Nacional, 2008), en su título II: Derechos, Sección Octava: Trabajo y Seguridad Social, articulo 33, que enuncia.

El trabajo es un derecho y un deber social, y un derecho económico, fuente de realización personal y base de la economía. El Estado garantizará a las personas trabajadoras el pleno respeto a su dignidad, una vida decorosa, remuneraciones y retribuciones justas y el desempeño de un trabajo saludable y libremente escogido o 168 
aceptado (p. 18). Asimismo, el Reglamento de Seguridad y Salud de los Trabajadores y Mejoramiento del Medio Ambiente de Trabajo (2008), artículo 11, numeral 2, asiente "adoptar las medidas necesarias para la prevención de los riesgos que puedan afectar la salud y el bienestar de los trabajadores en los lugares de trabajo de su responsabilidad" (p. 06). En la Ley Orgánica del Servicio Público (Asamblea Nacional, 2010), en adelante LOSEP en su artículo 25, establece como jornada especial aquella que por la misión que cumple la institución o sus servidores, no puede sujetarse a la jornada única y requiere de jornadas, horarios o turnos especiales; debiendo ser fijada para cada caso, observando el principio de continuidad, equidad y optimización del servicio, acorde a la norma que para el efecto emita el Ministerios de Relaciones Laborales (p. 17).

Se deja entrever, que existen diversas regulaciones para controlar y prevenir los riesgos y las condiciones inseguras e insalubres en el trabajo, generadoras de accidentes, lesiones y enfermedades profesionales u ocupacionales, las autoras consideraron importante realizar un bosquejo de las diferentes posturas legales y normativas al respecto, lo que permite al lector observar los diferentes mecanismos y por tanto la importancia que cobra cada vez más este tema en el trabajo, aun cuando la naturaleza de cada organización o institución es definida por una serie de actividades diferentes y cada una expuesta a riesgos de diferente índole y escala, algunas leves y otras de fuerte impacto en la salud.

Es por ello, que el objeto de estudio de la presente investigación referido a las enfermedades ocupacionales del personal de enfermería por desempeño de labores en turnos rotativos y la importancia de los protocolos ergonómicos de prevención, es un tema de gran interés para las autoras. Algunas de ellas, observadoras participantes, por formar parte de la institución que se estudia, les permite tener una visión amplia de los efectos de la cotidianidad laboral y las condiciones en que esta se desarrolla (Borges, 1998, p. 113). "Todos estos factores de riesgos, aunados a las condiciones y estilos de vida, configuran perfiles de malestares, enfermedades, desgaste físico y emocional, incapacidades e insatisfacción laboral, específicos del personal de enfermería." En el mismo orden de ideas, una de las principales condiciones a las que se encuentra expuesto el profesional de enfermería es la jornada por turno rotativo; tal es el caso de la población objeto de estudio, ubicada en el área 1 y 2 de Traumatología del Hospital Eugenio Espejo, quienes laboran en turnos rotativos de 12 horas diurnas, al siguiente día 12 horas nocturnas, con descanso de 72 horas repitiendo el ciclo de forma progresiva, en cada turno trabajan tres profesionales de enfermería que tienen bajo su responsabilidad aproximadamente 42 pacientes. Las actividades principales van desde la entrega y recepción de turno, preparación y suministro de medicación, control de signos vitales, peso, talla, control de ingesta y excreta, planificar el preoperatorio del paciente quirúrgico, hasta registrar en la historia clínica virtual los formularios por paciente. Tal como se explicó con anterioridad, estos horarios irrumpen en una serie de condiciones biológicas y naturales, alterando el ciclo natural del organismo y por consiguiente la salud del afectado, en la medida en que este se encuentre expuesto a estas desvinculaciones (Serra, 2013, p. 444).

No es el horario per se, el nocivo, sino su interacción con nuestro reloj biológico y que determina un concepto fundamental en relación al impacto del trabajo en turnos en el organismo que es el de la Cronodisrupción, es decir el desacople entre la fase 169 


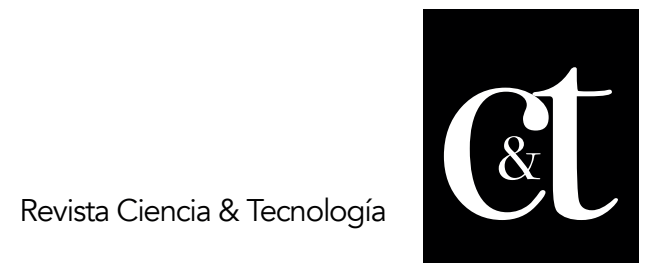

No. 20, 31 de octubre de 2018

ISSN impreso: 1390 - 6321

de los sistemas biológicos internos relativos al medio externo. Al mismo tiempo, se tiene el ciclo de vigilia del sueño, en el hipotálamo se encuentra un reloj corporal interno que regula los ritmos corporales, incluido el ritmo de vigilia- sueño. El período habitual de la mayoría de estos ritmos es cercano a 24 horas (circadiano) y se sincroniza con el ritmo ambiental diario de luz-oscuridad coincidiendo la fase de activación con las horas diurnas, y las de inhibición con las horas nocturnas (Serra, 2013).

Esto influye en las funciones corporales, que durante la noche se produce una disminución de las capacidades físicas y mentales, la memoria a largo plazo disminuye y mejora progresivamente a lo largo del día, en momentos del día la rapidez de nuestras respuestas es máxima, mientras que en otros producimos respuestas más lentas, otras áreas experimentan variaciones a lo largo de la jornada como el tono muscular, la frecuencia cardíaca, la temperatura corporal, la frecuencia respiratoria.

Por tanto, el trabajo nocturno esfuerza al organismo de la persona expuesta a invertir su ciclo normal de actividad y descanso, conjuntamente con la falta de sueño que suele asociarse a estas condiciones laborales, siendo responsable de diversos problemas de salud asociados al trastorno del sueño, síndrome de burnout, enfermedades cardiovasculares, enfermedades gastrointestinales, desordenes metabólicos, trastornos reproductivos, cáncer, dificultades en las relaciones sociales, familiares y laborales (Borges, 1998, p. 114).

En líneas generales, los factores de riesgos ocupacionales a los que se exponen los trabajadores de la salud, y en particular el personal de enfermería (por ser el colectivo de trabajadores y trabajadoras en contacto directo con los enfermos), pueden clasificarse en: factores de riesgos biológicos, factores de riesgos químicos, factores fisiológicos o de sobrecarga física, factores sanitarios, factores físicos, factores mecánicos o de riesgo de accidentes y factores psicosociales.

Con respecto al trastorno del sueño en el trabajador que labora en jornada nocturna, se produce cuando el horario laboral se desarrolla en el periodo de sueño habitual para el trabajador y por lo tanto no consigue adaptar su ritmo biológico al horario de vigilia-sueño, las deficiencias de sueño: insomnio y por otra parte la excesiva somnolencia durante el día y/o durante su turno de trabajo son predictores del desarrollo trastornos del estado de ánimo, ansiedad y depresión mayor (Sierra Delgado y Carretero, 2009).

Asimismo, el síndrome de Burnout se define como una respuesta al estrés laboral crónico. Comprende actitudes y sentimientos negativos hacia las personas con las que se trabaja y hacia el propio rol profesional, así como la vivencia de encontrarse emocionalmente agotado, generalmente en el turno de noche se obtiene un menor baja realización personal, estado que se ve agravado por un entorno laboral poco organizado y una menor calidad de trabajo realizado; factores que producen relaciones sociales y familiares de mala calidad (Cantos, Barbecho y Ochoa, 2014).

Existe evidencia de que el trabajo nocturno está asociado con mayor riesgo de enfermedades cardiovasculares, ya que los patrones de sueño irregulares causan fatiga y afectan adversamente las funciones fisiológicas. Se pueden observar 


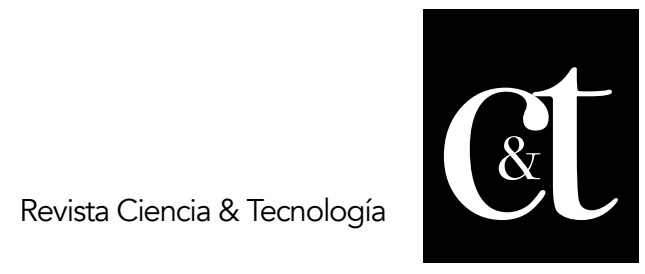

No. 20, 31 de octubre de 2018

ISSN impreso: 1390 - 6321

cambios en la frecuencia cardíaca, elevación de la presión arterial y disritmias, debido a la alteración de la excreción de catecolaminas, elevaciones del colesterol sérico, ácido úrico y potasio durante y después del turno de noche (Rauchenzauner et al., 2009).

También, el trabajo por turnos expone a los trabajadores a trastornos gastrointestinales debido a las modificaciones en hábitos alimenticios, horarios de comidas, el abuso del consumo de café, bebidas energizantes y tabaco por lo que suelen estar presentes flatulencias, ardor estomacal, ulceras digestivas, estreñimiento, problemas hemorroidales, diarreas por el escaso consumo de una dieta rica en fibra (Knutsson \& Boggild, 2010), tienen estudios que demuestran aumento de peso y obesidad parten de los hábitos alimenticios y de ejercicio; así como, la interrupción en el ritmo circadiano parece contribuir, observándose predisposición respecto a quienes trabajan por turnos y en horario nocturno, los mismos que aparecen como factores de riesgo independiente, estas apreciaciones aún se mantienen en estudio (Robin y Harvey, 2010).

Estudios recientes a nivel mundial han puesto de manifiesto una asociación entre el trabajo nocturno y un aumento del riesgo de cáncer de mama, ya que la exposición a la luz artificial nocturna inhibe la síntesis de melatonina aumentando el estímulo estrogénico e inhibiendo el efecto antimitótico, antiangiogénico y antioxidante de esta hormona, aumentando así el riesgo tumoral. La Agencia Internacional para la Investigación del Cáncer en el 2007 consideró el trabajo nocturno como un riesgo potencial que aumenta la probabilidad de generar cáncer de mama, colon-rectal y de pulmón (Fresneda, Gómez y Bascopé, 2013; Schernhammer, Feskanich, Liang, \& Han, 2013).

Finalmente, el trabajo a turnos imposibilita el mantener relaciones sociales o un momento de ocio en los horarios tradicionales, por lo que se presentaría aislamiento al no compartir suficiente tiempo de esparcimiento y relaciones con familia, amigos y compañeros de trabajo Los profesionales de enfermería trabajan durante la noche con jornadas de 12 horas consecutivas, no obstante en general no llegan a sus casas a descansar sino que dedican su tiempo a la realización de las actividades que se desprenden de su condición de género, como atender a sus hijos y a efectuar trabajo doméstica (García, 2013).

\section{Metodología}

El trabajo de investigación que se presenta a través de esta manuscrito, tiene como objetivo general estudiar las enfermedades ocupacionales del personal de enfermería que labora por turnos rotativos en el área de Traumatología del Hospital Eugenio Espejo con la finalidad de establecer mecanismos o protocolos ergonómicos para su prevención. Se enmarca en un enfoque cuantitativo, diseño de campo. Los datos se obtienen directamente del lugar donde ocurren los hechos; de tipo descriptivo porque no se influye en las variables, solo se observa la dinámica del objeto sin influir el ella.

Como técnica de recolección de los datos se diseña una encuesta a través de un cuestionario de 21 preguntas con opciones de respuesta múltiples, que se aplica a una población finita constituida por trece (13) profesionales de la enfermería que laboran en las áreas 1 y 2 de Hospitalización de Traumatología del Hospital de 171

Rueda, Toapanta, Andino, Rueda. Enfermedades ocupacionales del personal de enfermería por desempeño de labores en turnos rotativos y la importancia de los protocolos ergonómicos de prevención 


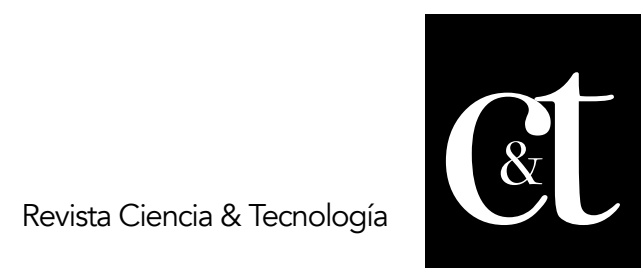

No. 20, 31 de octubre de 2018

ISSN impreso: 1390 - 6321

Especialidades Eugenio Espejo. El cuestionario se divide en tres importantes dimensiones: estado de salud, turnos nocturnos y cuestionario Maslach. Este es un cuestionario estandarizado para detectar expectaciones del Síndrome de Burnout y que consta de 22 preguntas dicotómicas cerradas que en conjunto valoran agotamiento emocional, despersonalización y baja realización personal.

Este instrumento de recolección de los datos fue validado a través del juicio de expertos que evaluaron el cuestionario a través de un formulario de validación, el cual arroja observaciones que fueron corregidas. Para poder aplicar la encuesta, esta, a su vez, fue sacada su confiabilidad en el formulario, considerando las categorías de pertinencia. Luego de ello, se procedió a entregar el cuestionario a cada participante, para tabular las respuestas dadas en anonimato, descargando los resultados en tablas de frecuencia con su respectivo grafico de barras, bajo el ambiente de Microsoft Excel 2016.

\section{Resultados y discusión}

En las áreas 1 y 2 de Hospitalización de Traumatología del Hospital de Especialidades Eugenio Espejo, se aplicó un cuestionario de 21 preguntas a trece (13) profesionales de enfermería que laboran en turnos rotativos y se encontraban activos al momento de realizar el estudio, de los cuales un $76.92 \%$ representado por mujeres y un $23.08 \%$ estaba representado por hombres, otro hallazgo encontrado es que el $62 \%$ de esta población se encontraba en edades comprendidas entre 26 a 35 años y un $31 \%$ entre 36 a 45 años, lo que implica que la mayoría del personal son adultos jóvenes situados en una edad activa.

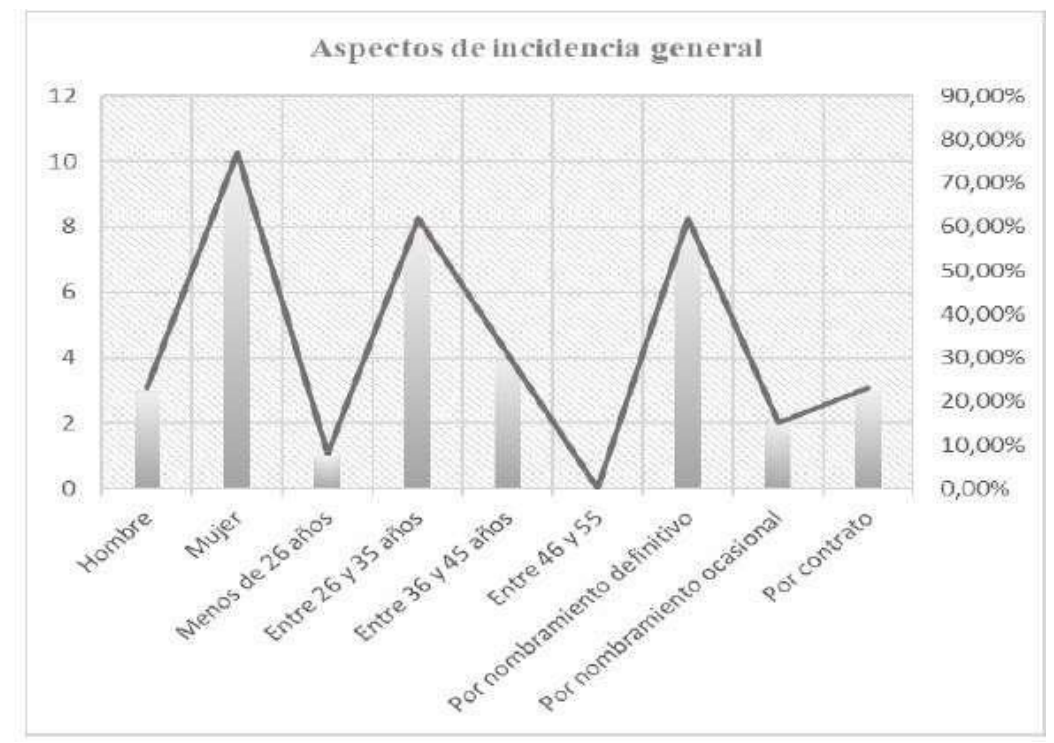

Gráfico 1. Aspectos generales de incidencia Fuente: Elaboración propia

Así mismo, el $62 \%$ es personal con nombramiento definitivo, es decir, tienen una relación laboral formal, legal y fija con el hospital, otro $23 \%$ es contratado, cuando se le realizo la pregunta que respondiera como definía su estado de salud en escala de Excelente a Mala, un $77 \%$ respondió que la consideraba buena (obviando el 


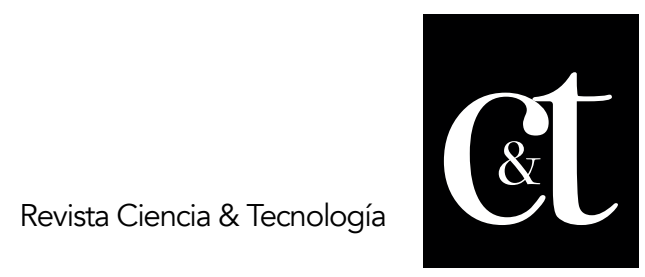

No. 20, 31 de octubre de 2018

ISSN impreso: 1390 - 6321

indicador excelente y muy buena), otro $15 \%$ respondió que excelente, sin embargo al preguntar si tenía dolencias en la espalda, un 31\% respondió que poseía malestares en la espalda con frecuencia alta, el resto también las poseía, pero con menos frecuencia (media o baja).

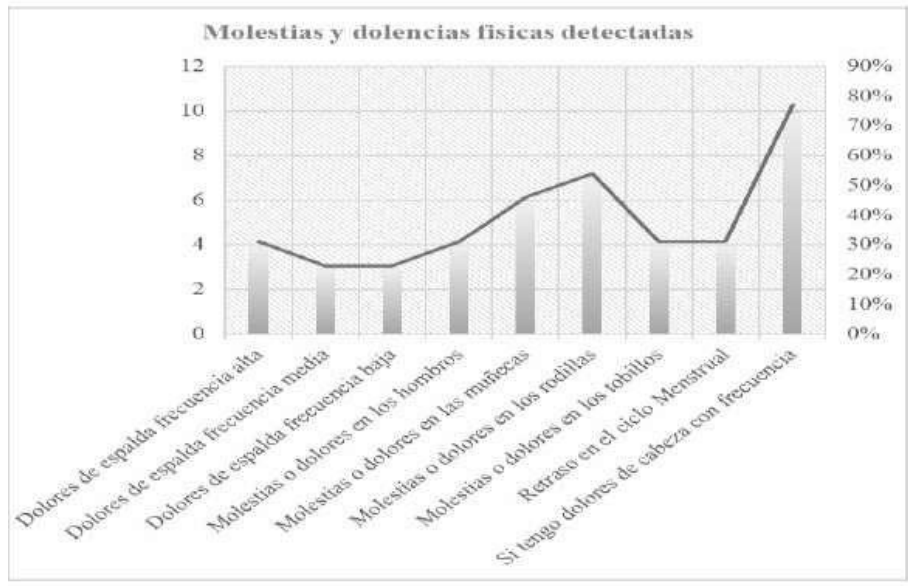

\section{Gráfico 2. Molestias y dolencias físicas detectadas}

Fuente: Elaboración propia

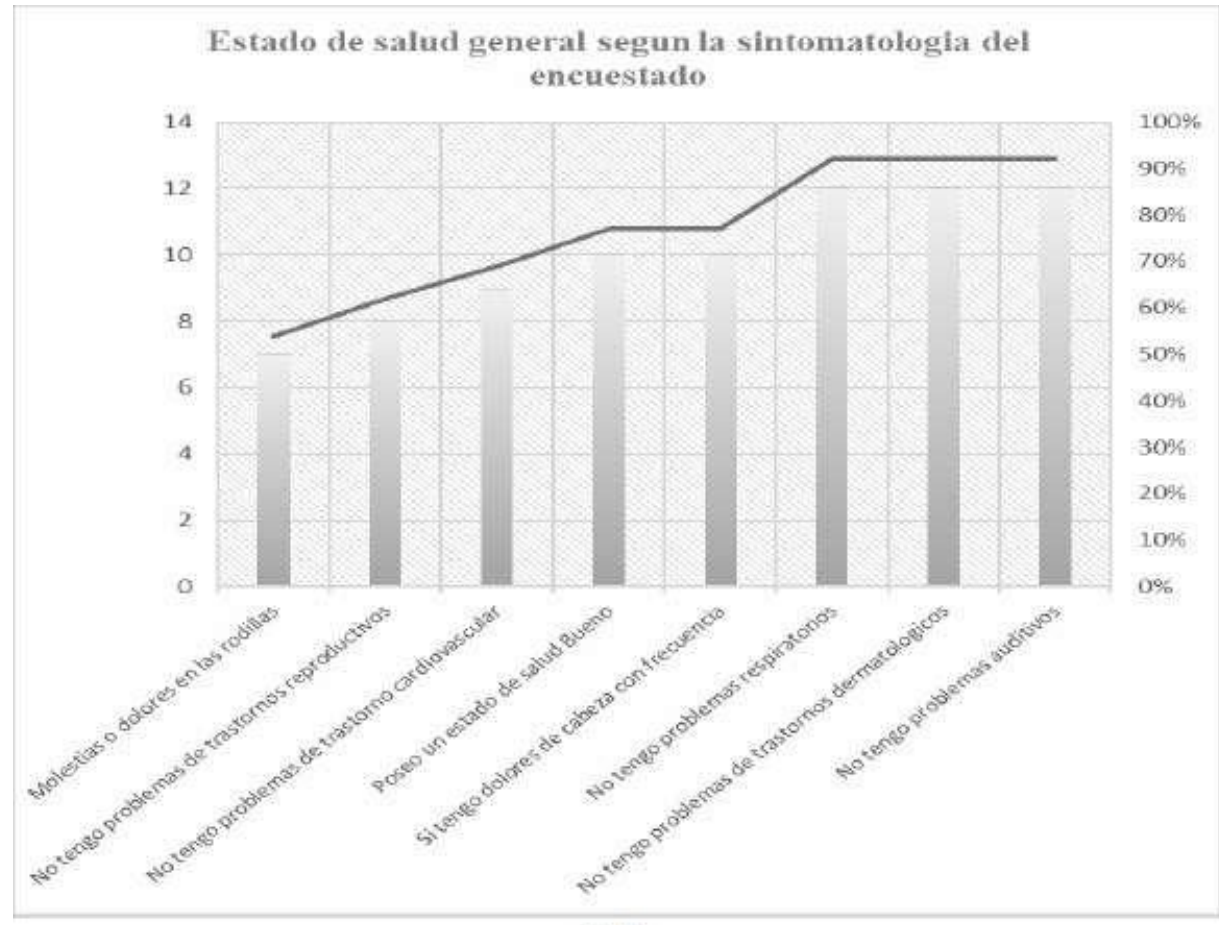

Gráfico 3 Estado general de salud, sēgún la sintomatología del encuestado Fuente: Elaboración propia

Cuando se preguntó sobre las lesiones producidas en los últimos 12 meses dentro de 


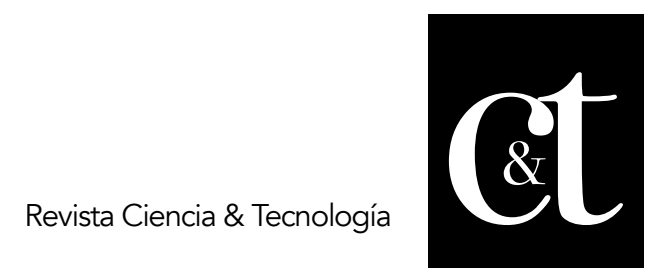

No. 20, 31 de octubre de 2018

ISSN impreso: 1390 - 6321

la jornada laboral, todos coincidieron en un rotundo no, sin embargo, la mayoría compuesta por un $62 \%$ afirma que no puede descansar cuando le corresponde el horario nocturno, el total de los encuestados afirma que no posee un lugar específico para el descanso, ese $62 \%$ coincide en que no descansa y el restante $38 \%$ afirma descansar por intervalos, un $76 \%$ afirma sentirse fatigado o muy fatigado en la jornada nocturna, sin embargo un $85 \%$ dice poseer tiempo para la ingesta de alimentos.

Por otro lado, pero no menos importante, se tiene la evaluación de las condiciones psicológicas, a través de la aplicación del Test de Maslach que nos permite determinar la frecuencia e intensidad del Síndrome de Burnout; una reacción negativa frente a la demanda que requiere el trabajo y las relaciones interpersonales en las que desarrollan su labor, presentando en cansancio o agotamiento emocional, la sensación de fatiga como consecuencia de la actividad laboral, un 38\% coincide entre la categoría alta a moderada, razón por la cual se encuentra presente el riesgo potencial de presentar cansancio emocional en la mayoría de los encuestados bajo un $61 \%$. Asimismo, la despersonalización caracterizada por distanciamiento emocional, indiferencia en relación a las actividades laborales, pudiendo llegar a ver otras personas como objeto, el $69 \%$ de los encuestados obtuvieron un riesgo alto y un $23 \%$ alcanzaron puntajes con riesgo moderado.

En términos generales, los resultados de la aplicación del test, evidencian que el $100 \%$ de los encestados no sufre en la actualidad de Síndrome de Burnout, sin embargo, alcanzaron puntajes altos en una o más de las categorías sin conseguir completarlos y pueden ya indicar la presencia de estrés, pero sin llegar al diagnóstico completo del síndrome.

En vista de los resultados obtenidos es importante establecer protocolos ergonómicos para la población objeto de estudio, considerando como ventaja que es un grupo de profesionales de la enfermería que se encuentran en una edad donde se pueden corregir algunas sintomatologías y lograr una completa recuperación física y psicológica, por tanto, es importante establecer hábitos con el apoyo de la institución donde se permita:

1. Dormir en una habitación obscura (usar persianas, cortinas)

2. Mantener la habitación silenciosa con una temperatura adecuada entre los $23^{\circ} \mathrm{C}$

3. La superficie de descanso debe ser cómoda

4. Evitar la ingesta de cafeína 4 horas antes de irse a dormir

5. Tener un espacio en el área laboral solo para el descanso

Respecto al protocolo de alimentación; para las raciones de media mañana y media tarde elegir sopas vegetales, ensaladas, frutas, yogur, sándwiches integrales, queso, huevo, nueces y té verde. Evitar consumir productos ricos en azúcar como refrescos, panadería (pan blanco), dulces y no comer entre la medianoche y las 06.00 horas, trate de comer al comienzo y al final del turno, ni alimentos y bebidas clasificados como bocadillos de baja calidad. Evitar las comidas pesadas que superen el $20 \%$ de la ingesta diaria de energía 1-2 horas antes del episodio de sueño diario principal. El día post turno tomar el desayuno para evitar despertar debido al hambre cuando tome un descanso y Mantener un estilo de vida saludable con ejercicio. 


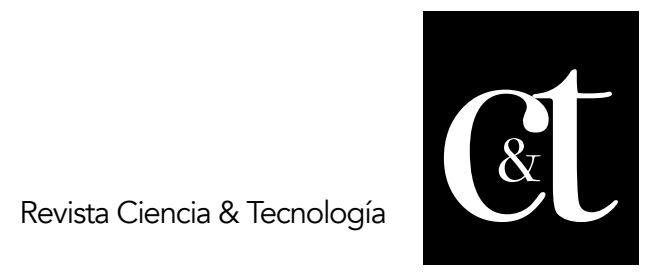

No. 20, 31 de octubre de 2018

ISSN impreso: 1390 - 6321

Finalmente es importante informar y concientizar al personal acerca de los turnos rotativos, las repercusiones en la salud y medidas preventivas, en temas como la fisiología del sueño normal y ritmos circadianos, la higiene del sueño y prevención de insomnio y socialización del protocolo de optimización del descanso, los estilos de vida saludable, socialización del protocolo de alimentación, las enfermedades relacionadas con las alteraciones del ritmo circadiano, la optimización del tiempo libre y comunicación familiar y el manejo del estrés.

\section{Conclusiones}

En el transcurso del desarrollo de esta investigación las autoras han confirmado a través la investigación de campo con apoyo de la revisión bibliográfica los efectos devastadores en la salud que posee el trabajo por turnos rotativos, lo cual implica consecuencias negativas tanto a nivel físico como psicológico en el personal de enfermería del Hospital Eugenio Espejo, área de Traumatología.

Respecto a las repercusiones de este sistema de trabajo en la salud de los trabajadores existen múltiples estudios que corroboran la aparición de enfermedades cardiovasculares, gastrointestinales, trastornos reproductivos, desórdenes metabólicos, cáncer; en el caso del personal de enfermería investigado, población adulta joven; en su gran mayoría mujeres reporta enfermedades cardiovasculares, trastornos reproductivos y se destaca un alto porcentaje de afectación osteomuscular; así como, dolores de cabeza, relacionamos esta alta incidencia de casos con la implementación desde el mes de Diciembre del 2016 del sistema informático HOSVITAL, con el objetivo de digitalizar la información relacionada con el paciente y por lo tanto al personal le toma 4 horas aproximadamente por turno realizando pausas para llenar todos los registros de enfermería, actividad que le lleva a cabo sentada frente al computador en un espacio estrecho sin acondicionamientos ergonómico.

Las repercusiones en el ámbito psicológico fueron evaluadas con el test de Maslach, encontrándose una alta presencia de cansancio emocional y despersonalización; sin embargo, existe un adecuado porcentaje de realización personal este último podría ser un factor protector ya que no hay casos de Síndrome de Burnout, a pesar de ello es necesario intervenir en el grupo sobre todo porque la despersonalización manifestada indica endurecimiento y deshumanización frente a las personas que atiende, incapacidad de empatía y sentimientos de no poder ayudar a los pacientes que necesitan ser atendidos.

Es necesario también un compromiso con la seguridad y salud de los trabajadores y cumplimiento de los requisitos legales de tal manera que se faciliten recursos materiales, técnicos y humanos para implementar un control integral de los riesgos a los que están expuestos el personal, y a su vez se estaría cumpliendo con las diferentes reglamentaciones que regulan la salud en el trabajo.

\section{Referencias bibliográficas}

Asamblea Nacional del Ecuador (2008). Constitución de la República del Ecuador. Montecristi. Recuperado

https://www.oas.org/juridico/pdfs/mesicic4 ecu const.pdf 


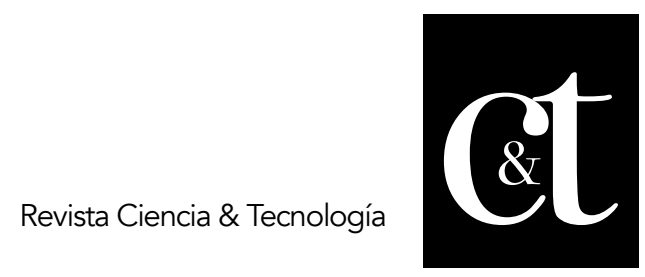

No. 20, 31 de octubre de 2018

ISSN impreso: 1390 - 6321

Asamblea Nacional del Ecuador (2010). Ley Orgánica del Servicio Público (LOSEP). Recuperado de https://www.educacionsuperior.gob.ec/wpcontent/uploads/downloads/2014/09/ LOSEP.pdf

Borges, A. (1998). Personal de Enfermería: Condiciones de trabajo de alto riesgo. Ponencia presentada en el marco de las III Jornadas Científicas de Enfermería "Txas Marina Reyes Alvarez". Revista Salud de los Trabajadores. Volumen 6. Recuperado de http://www.bvsde.ops-oms.org/bvsast/e/fulltext/personal/ personal.pdf

Cantos, M., Barbecho, M., y Ochoa, M. (2014). Prevalencia del síndrome de burnout en los profesionales de enfermería del servicio de emergencias del Hospital de Especialidades Eugenio Espejo. Recuperado de http://www.dspace.uce.edu.ec/bitstream/25000/4646/1/T-UCE-0006-69.pdf

Cuartero, P. (2002). Incidencia del mal descanso en el mundo laboral. XI Reunión anual de la Asociación Ibérica de Patología del Sueño (AIPS). Recuperado de http://www.vigilia-sueño.org/aips.asp

Fresneda, F., Gómez J. y Bascopé H. (2013). Riesgo de cáncer de mama en trabajadoras de turno nocturno. Revista de Medicina y Seguridad en el Trabajo, 146-158. Recuperado de http://www.ingentaconnect.com/content/doaj/0465546x/2013/00000059/0000 0230/art00010

García, M. (2013). Consecuencias psicofisiológicas del trabajo rotativo en la salud ocupacional del personal de enfermeras de la clínica alborada de Guayaquil. Universidad de Guayaquil Facultad de Ciencias Psicológicas. Recuperado de http://repositorio.ug.edu.ec/handle/redug/10399

Knutsson, A., \& Boggild, H. (2010). Gastrointestinal disorders among shift workers. Scandinavian Journal of Work, Environment \& Health Volume 36, No. 2. Recuperado de http://www.jstor.org/stable/40967835

Miró, E., Cano, M. y Buela, G. (2005). Sueño y Calidad de vida. Revista Colombiana de Psicología, Volumen 14, 11-27. Recuperado de https://revistas.unal.edu.co/index.php/psicologia/article/view/1215/1766

Organización Internacional del Trabajo - OIT (1990). Convenio 171. Recuperado de https://www.ilo.org/dyn/normlex/es/f?p=NORMLEXPUB:12100:0: :NO::P12100 _I NS TRUMENT_ID:312316

Rauchenzauner, M., Ernst, F., Hintringer, F., \& Ulmer, H. (2009). Arrhythmias and increased neuro-endocrine stress response during physicians' night shifts: A randomized cross-over trial. European Heart Journal 30(21):2606-2613.

Reglamento de Seguridad y Salud de los Trabajadores y Mejoramiento del Medio Ambiente de Trabajo (2017). Recuperado de http://www.trabajo.gob.ec/wp- 
content/uploads/downloads/2012/12/Reglamento-de-Seguridad-y-Salud-delos-Trabajadores-y-Mejoramiento-del-Medio-Ambiente-de-Trabajo-DecretoEjecutivo-2393.pdf

Schernhammer, E., Feskanich, D., Liang, G., \& Han, J. (2013). Rotating night-shift work and lung cancer risk among female nurses in the United States. Am J Epidemiol. 2013 Nov 1; 178(9): 1434-1441. Recuperado de https://www.ncbi.nlm.nih.gov/pmc/articles/PMC3813313/

Serra, L. (2013). Trabajo en turnos, privación de sueño y sus consecuencias clínicas y médicolegales. Revista Médica Clínica Las Condes, Volumen 24, 443-451. Recuperado https://www.sciencedirect.com/science/article/pii/S07168640137 01809

Sierra, J., C., Delgado, C. y Carretero H. (2009). Influencia de la calidad de sueño sobre variables psicopatológicas: un análisis comparativo entre trabajadores sometidos a turnos y trabajadores con horario normal. Revista Latinoamericana de Psicología, Volumen 41, Número 1. Bogotá, Colombia. 